\title{
More on the acute transient thyroid swelling following needle biopsy
}

\author{
Konstantinos Ziambaras
}

Endocrinologist

Dear Sir,

In response to the very interesting article that was published in Hormones 2012, 11(2): 147-150 “Acute transient thyroid swelling following needle biopsy: An update", I would like to share our experiences regarding this phenomenon. We have had six such cases of acute transient thyroid swelling during ultrasound guided fine needle aspiration (FNA). All six cases occurred within a relatively short period of time (a few months, making us wonder if there is a seasonal variation on this side effect). The swelling in all six cases was limited to the nodule and the surrounding area. No swelling was observed on the opposite lobe or the prethyroid tissues. There was no neck echymosis or airway obstruction and the swelling was, in most of the cases, painless. In fact, only one female patient complained of moderate pain which probably was exacerbated by her anxiety. It is worth mentioning that all patients were given local anesthesia with a sc lidocaine injection. It is also clear that these swellings were not caused by hemorrhage, which could be easily excluded by ultrasound. Moreover,

Key words: Acute transient swelling, Complications, Fine needle aspiration biopsy, Thyroid nodule

Address for correspondence:

Ziambaras Konstantinos, MD, PhD, Endocrinologist, 22 Arch. Makariou street and Skouladon, Iraklion, 71202, Crete, Greece, Tel.: +30-2810-282-678, E-mail: ziambaras@yahoo.gr Received 02-04-2013, Accepted 09-05-2013 all patients were instructed not to take any aspirin, NSAIDs or antiplatelet agents for at least one week before the procedure. It is also our experience that this complication resolves, although all our patients but one were given hydrocortisone IV. Fifteen to thirty minutes later the swelling had substantiallyalthough not completely - resolved and the patients were discharged without any further complication. A post FNA ultrasound was done in all six patients before they were discharged. This side effect does not appear to be age-dependent but it was more common in females, perhaps due to the fact that more women were submitted to this procedure. Nevertheless, our sample (of six patients) is rather too small to draw firm conclusions. At the beginning, we were definitely puzzled by this complication, since we were unable to explain it. Regarding the pathogenesis, our initial thoughts were that it was either some sort of idiosyncratic allergic reaction to the metallic needle or some kind of accidental contamination of the tissue via the needle with the gel we used for the probe. Finally, in one case in which we repeated the FNA six months later, we did not observe any repetition of this side effect. Interestingly, within the 10-year period during which FNAs have been performed in our private institute, we have encountered only these six cases, the last occurring almost 11 months ago.

\section{Sources of funding}

None

\section{Conflict of interest}

None 